\title{
SET MAPPINGS OF UNRESTRICTED ORDER
}

\author{
GREG G. GIBBON
}

\begin{abstract}
A set mapping on a set $S$ is a function $f$ mapping $S$ into the powerset of $S$ such that $x \notin f(x)$ for each $x$ in $S$. The set map $f$ has order $\theta$ if $\theta$ is the least cardinal such that $|f(x)|<\theta$ for each $x$ in $S$. A subset $H$ of $S$ is free for $f$ if $x \notin f(y)$ for all $x, y$ in $H$. In this paper we use classical results about set mappings of large order to investigate conditions which ensure a large free set for set mappings of unrestricted order.
\end{abstract}

A set mapping on a set $S$ is a function $f$ mapping $S$ into the powerset $P S$ of $S$ such that $x \notin f(x)$ for each $x \in S$. The set map is said to be of order $\theta$ if $\theta$ is the least cardinal such that $|f(x)|<\theta$ for each $x \in S$. A subset $H$ of $S$ is said to be free for $f$ if $x \notin f(y)$ for all $x, y \in H$.

A classical result of Hajnal [3] guarantees that a set mapping on a set of size $K$ and having order less than $K$ has a free set of size $K$. Set mappings of order at least equal to $k$ may have no non-trivial free sets. Consider the set mapping $f: K \rightarrow P K$ defined by $f(\alpha)=\{\beta \in K ; \beta<\alpha\}$. Clearly $f$ has order $k$, and yet there is no free set of size 2 .

Clearly extra conditions need to be imposed on a set mapping which has

Received 22 June 1983. The author wishes to thank Dr Neil H. Williams for his invaluable guidance in the preparation of this paper.

Copyright clearance Centre, Inc. Serial-fee code: 0004-9727/83 $\$ A 2.00+0.00$. 
order at least $K$, and certain intersection conditions were introduced by Erdös and Fodor [1]. Erdös, Hajnal and Rado [2] contains an exhaustive examination of the free set question with respect to set mappings of order equal to $k$ and satisfying these intersection conditions. They remark, in conclusion, that the question for set maps of order $k^{+}$(that is, unrestricted) appears to be equivalent to problems involving partition relations. In this paper we use methods that do not involve partition relations, and in one case our results are stronger than would seem to follow from partition relations.

Our notation will be conventional. We use $K$ for infinite cardinal numbers and $\lambda, \eta, \theta$ for cardinal numbers, finite or infinite. Other lower case Greek letters will denote ordinal numbers. The cardinal numbers are identified with the initial ordinals in the usual way. We denote the cardinal successor of $\kappa$ by $\kappa^{+}$, the cofinality of $\kappa$ by $\kappa^{\prime}$ and $\kappa$ is said to be regular if $\kappa^{\prime}=\kappa$, otherwise singular. Let $[A]^{\eta}$ denote the set $\{B \subseteq A ;|B|=\eta\}$ and $f[X]=\{f(x) ; x \in X\}$. Set mappings will always be defined on the set $K$.

DEFINITIONS. $f$ is said to satisfy the intersection condition $C(n, \theta)$ if

$$
x \in[\kappa]^{\eta} \Rightarrow|n f[X]|<\theta .
$$

The relation $(\kappa, n, \theta) \rightarrow \lambda$ means that whenever $f$ is a set mapping on $\kappa$ of order at most $k$ and satisfying $C(n, \theta)$, there is a $\lambda$ sized subset $F$ of $k$ which is free for $f$.

The relation $[\kappa, \eta, \theta] \rightarrow \lambda$ means that whenever $f$ is a set mapping on $\kappa$ satisfying $C(\eta, \theta)$, there is a $\lambda$ sized subset $F$ of $\kappa$ free for $f$.

The relation $[\kappa, \eta, \theta]^{R} \rightarrow \lambda$ means that whenever $f$ is a set mapping on $\kappa$ satisfying $C(n, \theta)$ and the condition $(\forall \alpha<\kappa)(f(\alpha) \cap \alpha=\emptyset)$, there is a $\lambda$ sized subset $F$ of $\kappa$ free for $f$.

A set mapping $f$ on $\kappa$ of order at most $\kappa$ and satisfying $C(n, \theta)$ is said to satisfy $(\kappa, \eta, \theta)$, and similarly for $[\kappa, \eta, \theta]$ and $[\kappa, \mathrm{n}, \theta]^{R}$. 
The following is easily seen to hold from the definitions.

LEMMA 1. Let $\lambda_{1} \leq \lambda, \eta_{1} \leq n$ and $\theta_{1} \leq \theta$. If $(\kappa, n, \theta) \rightarrow \lambda$ holds, then $\left(\kappa, \eta_{1}, \theta_{1}\right) \rightarrow \lambda_{1}$ holds, and the corresponding result holds for $[\kappa, \eta, \theta]$ and $[\kappa, \eta, \theta]^{R}$.

We summarize the well known results about the relation $(\kappa, \eta, \theta) \rightarrow \lambda$ (see, for example, Hajnal and Máté [4] or Williams [5]).

THEOREM 1. Let $n, \lambda, \theta^{+}<\kappa$. Then we have

(a) $(\kappa, \eta, \lambda) \rightarrow \kappa$ when $\kappa$ is singular;

(b) $(\kappa, \kappa, \theta)+\kappa$ when $k$ is regular;

(c) $\left(\kappa^{+}, \kappa^{+}, \kappa^{\prime}\right) \rightarrow \kappa^{\prime}$;

(d) $(\kappa, \kappa, 1)+\left(\kappa^{\prime}\right)^{+}$when $\kappa$ is singular;

(e) $\left(\kappa^{+}, 2, \kappa\right) \rightarrow \kappa^{+}$when $k$ is regular.

The main result is Theorem 2 .

THEOREM 2. Let $\kappa$ be an infinite cardinal. Then $(\kappa, \eta, \theta) \rightarrow \lambda$ holds if and only if $[\kappa, \theta, n]^{R} \rightarrow \lambda$ holds.

Proof. Suppose first that $(\kappa, \eta, \theta) \rightarrow \lambda$ holds. Let $f$ be a set mapping on $k$ which satisfies $[\kappa, \theta, \eta]^{R}$. We will show that $f$ has a free set of size $\lambda$.

Define a set mapping $\bar{f}: k \rightarrow P K$ by

$$
\bar{f}(\alpha)=\{\beta \in \kappa ; \alpha \in f(\beta)\} \text { for } \alpha<\kappa \text {. }
$$

Hence, for all $x, y \in \kappa$, we have $x \in f(y) \Leftrightarrow y \in \bar{f}(x)$, and so $F$ is free for $f$ if and only if $F$ is free for $\bar{f}$.

Further, $f$ satisfies $C(\theta, \eta) \Leftrightarrow \bar{f}$ satisfies $C(\eta, \theta)$. For, suppose $f$ satisfies $C(\theta, n)$ and let $X \in[\kappa]^{\eta} ;$ put $y=n \bar{f}[X]$. Then $X \subseteq n f[Y]$, since if $x \in X$ then $y \in \bar{f}(x)$ for all $y \in Y$ and so $x \in f(y)$ for all $y \in Y$. Hence, if $|y| \geq \theta$, then $|\cap f[Y]|<\eta$, since $f$ satisfies $C(\theta, \eta)$. This contradicts the fact that $|x|=n$, so $|y|<\theta$ and hence $\bar{f}$ satisfies $C(\eta, \theta)$. The converse follows similarly. Also, since, for $\alpha, \beta<\kappa, \alpha \in f(\beta) \Leftrightarrow \beta \in \bar{f}(\alpha)$, we have that 


$$
(\forall \alpha<\kappa)(f(\alpha) \cap \alpha=\emptyset) \Leftrightarrow(\forall \alpha<\kappa)(\bar{f}(\alpha) \subseteq \alpha) .
$$

Hence $\bar{f}$ satisfies $(\forall \alpha<k)(f(\alpha) \subseteq \alpha)$ and so has order less than or equal to $\kappa$.

Hence we see that $\bar{f}$ satisfies $(\kappa, n, \theta)$ and so by the hypothesis, $\bar{f}$ has a free set $F$ of size $\lambda$, which is free for $f$, so the result holds.

Now suppose $k$ is regular, and suppose that $[\kappa, \theta, n]^{R} \rightarrow \lambda$ holds. Suppose that $f$ is a set mapping on $\kappa$ which satisfies $(\kappa, \eta, \theta)$. We shall show that $f$ has a free set of size $\lambda$. Define $f_{0}: k \rightarrow E k$ and $f_{1}: K \rightarrow P K$ by

$$
f_{0}(\alpha)=f(\alpha) \cap \alpha \text { for all } \alpha<k
$$

and

$$
f_{1}(\alpha)=f(\alpha)-\alpha \text { for all } \alpha<\kappa
$$

Since $f_{1}(\alpha) \cap \alpha=\emptyset$ for all $\alpha<k$ and $f_{1}$ has order less than or equal to $K$, then since $K$ is regular it is easy to construct a set $G$ of size $\kappa$ that is free for $f_{1}$. Simply choose inductively ordinals $\beta_{\gamma}$ for all $\gamma<\kappa$ such that

$$
\beta_{\gamma}>\cup\left\{\cup f_{I}\left(\beta_{\alpha}\right) ; \alpha<\gamma\right\} \cup \cup\left\{\beta_{\alpha} ; \alpha<\gamma\right\} \text {. }
$$

Then $G=\left\{\beta_{\gamma} ; \gamma<k_{\}}\right.$is free for $f_{1}$, since for $\alpha<\gamma<\kappa, B_{\alpha}<\beta_{\gamma}$ and $\beta_{\gamma} \in f\left(\beta_{\alpha}\right)$ by construction, and $\beta_{\alpha} \neq f\left(\beta_{\gamma}\right)-\beta_{\gamma}$ from above. Define a set mapping $h: G \rightarrow P G$ by $h(\alpha)=f_{0}(\alpha) \cap G$ for all $\alpha \in G$. Clearly $h$ satisfies $C(n, \theta)$, since $f$ does, and if $\alpha \in h(\beta)$, then $\alpha<\beta$, as for $f_{0}$. Since $G$ has order type equal to $\kappa, G$ can be identified with $\kappa$ while preserving its natural ordering, and so $h$ can be regarded as a set mapping $h: \kappa \rightarrow P K$ satisfying $(\forall \alpha<\kappa)(h(\alpha) \subseteq \alpha)$ and satisfying $C(\eta, \theta)$. Hence it follows that the induced set mapping $\bar{h}: \kappa \rightarrow P K$ satisfies $[\kappa, \theta, \eta]^{R}$. By the hypothesis, there is a set of size $\lambda$ free for $\bar{h}$, and hence free for $h$. By identifying $k$ with $G$, it follows that there is a subset $H$ of $G$ of size $\lambda$ free for $h$, 
and hence free for $f$ since $x, y \in H \Rightarrow x \xi h(y)$ and $y \notin h(x) \Rightarrow x \notin f_{0}(y)$ and $y \notin f_{0}(x)$ (since $\left.H \subseteq G\right) \Rightarrow x \notin f(y)$ and $y \notin f(x)$. Thus $(\kappa, n, \theta) \rightarrow \lambda$ holds.

Suppose $\lambda$ is singular, and that $[\kappa, \theta, \eta]^{R} \rightarrow \lambda$ holds. We shall prove that $(\kappa, \eta, \theta) \rightarrow \lambda$ holds by examining three cases.

Case (a). $\theta=k$. Trivial, as the hypothesis is false. (For example, put $f(\alpha)=\{\beta<\kappa ; \alpha<\beta\}$ for all $\alpha<\kappa$.)

Case (b). $\theta<\kappa$ and $\eta<\kappa$. Trivial, since by the results of Theorem 1 ( $a$ ), the result is true for all cardinals $\lambda$ with $\lambda \leq k$.

Case (c). $\theta<\kappa$ and $\eta=\kappa$. For $\lambda \leq \kappa^{\prime}$, the result is true as in Case (b). For $\lambda \geq\left(k^{\prime}\right)^{+}$, the result is false (see Theorem $l(d)$ ), and so it remains to show that the hypothesis is false. We shall use the following standard construction. Take an increasing sequence of cardinals $\left\{\kappa_{\sigma} ; \sigma<\kappa^{\prime}\right\}$ such that each $\kappa_{\sigma}$ is a successor cardinal and $\kappa=\Sigma\left(\kappa_{\sigma} ; \sigma<\kappa^{\prime}\right)$. For $\sigma<\kappa^{\prime}$, put $S_{\sigma}=\kappa_{\sigma}-U\left\{\kappa_{\beta} ; \beta<\sigma\right\}$, so the union $K=U\left\{S_{\sigma} ; \sigma<K^{\prime}\right\}$ is pairwise disjoint and each $S_{\sigma}$ is of size $\kappa_{\sigma}$. Define $f: \kappa \rightarrow P K$ by $f(\alpha)=S_{\sigma} \cap \alpha$ where $\alpha \in S_{\sigma}$. If $X$ is a set in $[\kappa]^{K}$, then $X$ must meet more than one of the sets $S_{\sigma}$, and so $n f[X]=\emptyset$. Also, if $S^{\prime}$ is a subset of $k$ free for $f$, then for each $\sigma<K^{\prime}$ clearly $\left|S^{\prime} \cap S_{\sigma}\right| \leq 1$ and hence $\left|S^{\prime}\right| \leq K^{\prime}$. So $f$ has no free set of size $\left(\kappa^{\prime}\right)^{+}$, and this example satisfies $(\kappa, \kappa, 1)$. Noting that $f$ also satisfies $(\forall \alpha<\kappa)(f(\alpha) \subseteq \alpha)$, we see that $\bar{f}$ satisfies $[\kappa, l, \kappa]^{R}$ and has no free set of size $\left(\kappa^{\prime}\right)^{+}$. This shows $[\kappa, 1, \kappa]^{R} \nrightarrow\left(\kappa^{\prime}\right)^{+}$, and so $[\kappa, \theta, \kappa]^{R} \nrightarrow \lambda$ for $\theta<\kappa$ and $\left(\kappa^{\prime}\right)^{+} \leq \lambda$.

The following symmetry lemma will simplify the analysis of the symbol $[\kappa, \eta, \theta] \rightarrow \lambda$. .

LEMMA 2. For all cardinals $\kappa, n, \theta, \lambda,[\kappa, n, \theta] \rightarrow \lambda$ holds if and only if $[\kappa, \theta, \eta] \rightarrow \lambda$ holds.

Proof. Suppose $[\kappa, n, \theta] \rightarrow \lambda$ holds, and let $f$ be a set mapping satisfying $[\kappa, \theta, n]$. We will show that $f$ has a free set of size $\lambda$. 
As in the proof of Theorem 2, $\bar{f}$ satisfies $[\kappa, n, \theta]$ and so by hypothesis has a free set of size $\lambda$ which is then free for $f$, and the result follows. The proof of the converse is identical.

In view of Lemma 2 the analysis of the relation $[\kappa, \eta, \theta] \rightarrow \lambda$ for a limit cardinal $k$ is completed by the following.

THEOREM 3. Let $\kappa$ be a limit cardinal and $\eta, \theta<\kappa$. Then

(a) $[\kappa, k, 1] \nrightarrow 2$,

(b) $[\kappa, n, \theta] \rightarrow \kappa$.

Proof. (a) Define $f: \kappa \rightarrow P k$ by $f(\alpha)=\kappa-(\alpha+1)$. Clearly $f$ satisfies $[\kappa, \kappa, 1]$, but has no free set of size 2 .

(b) Suppose that $f$ is set mapping satisfying $[\kappa, n, \theta]$. Define set mappings $f_{0}, f_{1}$ by

$$
\begin{aligned}
& f_{0}(\alpha)=f(\alpha) \cap \alpha, \\
& f_{1}(\alpha)=f(\alpha)-\alpha .
\end{aligned}
$$

Clearly $f_{0}$ satisfies $C(\eta, \theta)$ and $(\forall \alpha<\kappa)\left(\left|f_{0}(\alpha)\right|<\kappa\right)$ and so satisfies $(\kappa, \eta, \theta)$. Using Theorem $I(a)$ or $I(b),(\kappa, \eta, \theta) \rightarrow \kappa$ holds and so there is a set $F$ in $[\kappa]^{K}$ free for $f_{0}$.

Clearly $f_{1}$ satisfies $C(\eta, \theta)$ and $(\forall \alpha<\kappa)\left(f_{1}(\alpha) \cap \alpha=\varnothing\right)$ and hence satisfies $[\kappa, n, \theta]^{R}$. Defining $h: f \rightarrow P F$ by $h(\alpha)=f_{1}(\alpha) \cap F$ and noting that the order type of $F$ equals $K$, it is easy to see that, as in the proof of Theorem $2, h$ satisfies $[\kappa, \eta, \theta]^{R}$. Again applying Theorem I, $(\kappa, \theta, n) \rightarrow \kappa$ holds and so $[\kappa, n, \theta]^{R} \rightarrow \kappa$ follows from Theorem 2. Hence $h$ has a free set $G$ in $[F]^{K}$. Clearly $G$ is free for $f_{0}$ and hence for $f$.

For successor cardinals $K$ we have the following results.

THEOREM 4. For $\theta<\lambda$ and $\mathrm{k}=\lambda^{+}$,

(a) $[k, \theta, \theta] \rightarrow K$,

(b) $[k, k, 1] \nrightarrow 2$, 
(c) $[\kappa, \lambda, 1] \rightarrow \kappa$,

(d) $[\kappa, \lambda, 2] \nrightarrow k$ when $\lambda$ is regular,

(e) $[\kappa, \lambda, \theta] \rightarrow \lambda^{\prime}$.

Proof. (a) Similar to the proof of Theorem 3 (b), using the result $(\kappa, \kappa, \theta) \rightarrow \kappa$ from Theorem $1(b)$.

(b) As in Theorem 3 (a).

(c) Using symmetry, it suffices to show $[\kappa, 1, \lambda] \rightarrow \kappa$, and this follows trivially since $C(I, \lambda)$ implies that the order of $f$ is less than $K$.

(d) Follows from symmetry and the result $(\kappa, 2, \lambda) \nrightarrow \kappa$ from Theorem I $(e)$.

(e) Similar to the proof of Theorem $3(b)$, using the result $(\kappa, \kappa, \theta) \rightarrow \kappa$ to ensure a free set $F$ of size $\kappa$ for $f_{0}$, and using the result $(\kappa, \kappa, \lambda) \rightarrow \lambda^{\prime}$ from Theorem 1 to ensure a free set of size $\lambda^{\prime}$ in $E$ for $f_{1}$.

Theorem 4 leaves unsettled the question as to what size free set is guaranteed for a set map satisfying $[k, \lambda, \lambda]$ when $\lambda$ is regular. Clearly from Theorem $4(d)$ we have $[k, \lambda, \lambda] \rightarrow k$, but this theorem does not answer the question of the truth of the relation $[\kappa, \lambda, \lambda] \rightarrow \lambda$.

For singular $\lambda$, Theorem 4 leaves open a number of questions. When a set mapping satisfies $[\kappa, \lambda, \theta]$, Theorem 4 guarantees a free set of size $\lambda^{\prime}$ but does not answer whether $[\kappa, \lambda, \theta] \rightarrow \eta$ is true for $\left(\lambda^{\prime}\right)^{+} \leq n \leq \kappa$. Further, the truth of the relation $[\kappa, \lambda, \lambda] \rightarrow \eta$ is left open for all non-trivial $n$.

\section{References}

[1] P. Erdös and G. Fodor, "Some remarks on set theory - V", Acta Sci. Math. (Szeged) 17 (1957), 250-260.

[2] P. Erdös, A. Hajnal and R. Rado, "Partition relations for cardinal numbers", Acta Math. Acad. Sci. Hungar 16 (1965), 93-196. 
[3] A. Hajnal, "Proof of a conjecture of S. Ruziewicz", Fund. Math. 50 $(1961 / 62), 123-128$.

[4] A. Hajnal and A. Mate, "Set mappings, partitions, and chromatic numbers", Logic ColZoquium, 1973, 347-379 (North Holland, Amsterdam, 1975).

[5] Neil H. Williams, Combinatorial set theory (Studies in Logic and the Foundations of Mathematics, 91. North Holland, Amsterdam, New York, Oxford, 1977).

Department of Mathematics,

University of Queensland,

St Lucia,

Queensland 4067,

Australia. 